\title{
The geometric classification of Leibniz algebras
}

\author{
Nurlan Ismailov $^{a}$, Ivan Kaygorodov ${ }^{b}$, Yury Volkov ${ }^{c}$ \\ ${ }^{a}$ Suleyman Demirel University, Almaty, Kazakhstan \\ ${ }^{b}$ Universidade Federal do ABC, CMCC, Santo André, Brazil. \\ ${ }^{c}$ Saint Petersburg State University, Saint Petersburg, Russia. \\ E-mail addresses: \\ Nurlan Ismailov (nurlan.ismail@gmail.com), \\ Ivan Kaygorodov (kaygorodov.ivan@gmail.com), \\ Yury Volkov (wolf86_666@list.ru).
}

\begin{abstract}
We describe all rigid algebras and all irreducible components in the variety of four dimensional Leibniz algebras $\mathfrak{L e i b}_{4}$ over $\mathbb{C}$. In particular, we prove that the Grunewald-O'Halloran conjecture is not valid and the Vergne conjecture is valid for $\mathfrak{L} \mathfrak{e} \mathfrak{i b}_{4}$.

Keywords: Leibniz algebra, Grunewald-O'Halloran conjecture, Vergne conjecture, orbit closure, degeneration, rigid algebra
\end{abstract}

2010 MSC: 14D06, 14L30.

\section{INTRODUCTION}

Degenerations of algebras is an interesting subject, which was studied in various papers (see, for example, [3, 4, 7, 9, 10, 14, 16, 19, 23]). In particular, there are many results concerning degenerations of algebras of low dimensions in a variety defined by a set of identities. One of important problems in this direction is the description of so-called rigid algebras. These algebras are of big interest, since the closures of their orbits under the action of generalized linear group form irreducible components of a variety under consideration (with respect to the Zariski topology). For example, the rigid algebras were classified in the varieties of two dimensional bicommutative algebras in [14], three dimensional Novikov algebras in [3], three dimensional Leibniz in [22], four dimensional Lie algebras in [4], four dimensional Jordan algebras in [17], four dimensional Zinbiel algebras and nilpotent four dimensional Leibniz algebras in [16], unital five dimensional associative algebras in [19], nilpotent five- and sixdimensional Lie algebras in [9,23], nilpotent five- and six-dimensional Malcev algebras in [15], and some other.

The Leibniz algebras were introduced as a generalization of Lie algebras. The study of the structure theory and other properties of Leibniz algebras was initiated by Loday in [18]. Leibniz algebras were also studied in [6, 8, 20]. An algebra $A$ is called a Leibniz algebra if it satisfies the identity

$$
(x y) z=(x z) y+x(y z) \text {. }
$$

It is easy to see that any Lie algebra is a Leibniz algebra. At this moment, the algebraic classification of $n$ dimensional Leibniz algebras over $\mathbb{C}$ is known only for $n \leq 4$.

\footnotetext{
${ }^{1}$ The work was supported by RFBR 17-01-00258 and by the President's Program "Support of Young Russian Scientists" (grant MK-1378.2017.1).
} 
In this paper we describe all rigid algebras and all irreducible components in variety of $\mathfrak{L e i b}_{4}$. As a result, we show that $\mathfrak{L e i b}_{4}$ has 5 rigid algebras and 16 irreducible components.

\section{DEFINITIONS AND NOTATION}

All spaces in this paper are considered over $\mathbb{C}$, and we write simply $\operatorname{dim}, \mathrm{Hom}$ and $\otimes$ instead of $\operatorname{dim}_{\mathbb{C}}, H o m_{\mathbb{C}}$ and $\otimes_{\mathbb{C}}$. An algebra $A$ is a set with a structure of a vector space and a binary operation that induces a bilinear map from $A \times A$ to $A$.

Given an $n$-dimensional vector space $V$, the set $\operatorname{Hom}(V \otimes V, V) \cong V^{*} \otimes V^{*} \otimes V$ is a vector space of dimension $n^{3}$. This space has a structure of the affine variety $\mathbb{C}^{n^{3}}$. Indeed, let us fix a basis $e_{1}, \ldots, e_{n}$ of $V$. Then any $\mu \in \operatorname{Hom}(V \otimes V, V)$ is determined by $n^{3}$ structure constants $c_{i, j}^{k} \in \mathbb{C}$ such that $\mu\left(e_{i} \otimes e_{j}\right)=\sum_{k=1}^{n} c_{i, j}^{k} e_{k}$. A subset of $\operatorname{Hom}(V \otimes V, V)$ is Zariski-closed if it can be defined by a set of polynomial equations in the variables $c_{i, j}^{k}(1 \leq i, j, k \leq n)$.

All algebra structures on $V$ satisfying Leibniz identity form a Zariski-closed subset of the variety $\operatorname{Hom}(V \otimes V, V)$. We denote this subset by $\mathfrak{L e i b}_{n}$. The general linear group $G L(V)$ acts on $\mathfrak{L e i b}_{n}$ by conjugations:

$$
(g * \mu)(x \otimes y)=g \mu\left(g^{-1} x \otimes g^{-1} y\right)
$$

for $x, y \in V, \mu \in \mathfrak{L e i b}_{n} \subset \operatorname{Hom}(V \otimes V, V)$ and $g \in G L(V)$. Thus, $\mathfrak{L e i b}_{n}$ is decomposed into $G L(V)$-orbits that correspond to the isomorphism classes of algebras. Let $O(\mu)$ denote the orbit of $\mu \in \mathfrak{L e i b}_{n}$ under the action of $G L(V)$ and $\overline{O(\mu)}$ denote the Zariski closure of $O(\mu)$.

Let $A$ and $B$ be two $n$-dimensional Leibniz algebras and $\mu, \lambda \in \mathfrak{L e i b}_{n}$ represent $A$ and $B$ respectively. We say that $A$ degenerates to $B$ and write $A \rightarrow B$ if $\lambda \in \overline{O(\mu)}$. Note that in this case we have $\overline{O(\lambda)} \subset \overline{O(\mu)}$. Hence, the definition of a degeneration does not depend on the choice of $\mu$ and $\lambda$. We write $A \not \rightarrow B$ if $\lambda \notin \overline{O(\mu)}$.

Let $A$ be represented by $\mu \in \mathfrak{L} \mathfrak{e i \mathfrak { b } _ { n }}$. Then $A$ is rigid in $\mathfrak{L} \mathfrak{e i b} \mathfrak{b}_{n}$ if $O(\mu)$ is an open subset of $\mathfrak{L e i b}_{n}$. Recall that a subset of a variety is called irreducible if it cannot be represented as a union of two non-trivial closed subsets. A maximal irreducible closed subset of a variety is called irreducible component. It is well known that any affine variety can be represented as a finite union of its irreducible components in a unique way. In particular, $A$ is rigid in $\mathfrak{L} \mathfrak{e} i \mathfrak{b}_{n}$ iff $\overline{O(\mu)}$ is an irreducible component of $\mathfrak{L e i b}_{n}$. This is a general fact about algebraic varieties whose proof can be found, for example, in [16].

We use the following notation:

(1) $\operatorname{Ann}_{L}(A)=\{a \in A \mid x a=0$ for all $x \in A\}$ is the left annihilator of $A$;

(2) $A n n_{R}(A)=\{a \in A \mid a x=0$ for all $x \in A\}$ is the right annihilator of $A$;

(3) $\operatorname{Ann}(A)=A n n_{R}(A) \cap A n n_{L}(A)$ is the annihilator of $A$;

(4) $A^{(+2)}$ is the space $\{x y+y x \mid x, y \in A\}$.

Given spaces $U$ and $W$, we write simply $U>W$ instead of $\operatorname{dim} U>\operatorname{dim} W$. We write $U W$ ( $U, W \subset V$ ) for the product of subspaces of $V$ with respect to the multiplication $\mu$. We use the notation $S_{i}=\left\langle e_{i}, \ldots, e_{4}\right\rangle, i=1, \ldots, 4$. 


\section{Methods}

In the present work we use the methods applied in our previous works (see [14-16]).

To prove degenerations, we will construct families of matrices parametrized by $t$. Namely, let $A$ and $B$ be two Leibniz algebras represented by the structures $\mu$ and $\lambda$ from $\mathfrak{L e i b}_{n}$ respectively. Let $e_{1}, \ldots, e_{n}$ be a basis of $V$ and $c_{i, j}^{k}(1 \leq i, j, k \leq n)$ be the structure constants of $\lambda$ in this basis. If there exist $a_{i}^{j}(t) \in \mathbb{C}\left(1 \leq i, j \leq n, t \in \mathbb{C}^{*}\right)$ such that $E_{i}^{t}=\sum_{j=1}^{n} a_{i}^{j}(t) e_{j}(1 \leq i \leq n)$ form a basis of $V$ for any $t \in \mathbb{C}^{*}$, and the structure constants of $\mu$ in the basis $E_{1}^{t}, \ldots, E_{n}^{t}$ are such polynomials $c_{i, j}^{k}(t) \in \mathbb{C}[t]$ that $c_{i, j}^{k}(0)=c_{i, j}^{k}$, then $A \rightarrow B$. In this case $E_{1}^{t}, \ldots, E_{n}^{t}$ is called a parametrized basis for $A \rightarrow B$. It is easy to see that any algebra degenerates to the algebra with zero multiplication.

Let now $A(*):=\{A(\alpha)\}_{\alpha \in I}$ be a set of algebras, and let $B$ be another algebra. Suppose that, for $\alpha \in I, A(\alpha)$ is represented by the structure $\mu(\alpha) \in \mathfrak{L e i b}_{n}$ and $B \in \mathfrak{L e i b}_{n}$ is represented by the structure $\lambda$. Then $A(*) \rightarrow B$ means $\lambda \in \overline{\{O(\mu(\alpha))\}_{\alpha \in I}}$, and $A(*) \not \rightarrow B$ means $\lambda \notin \overline{\{O(\mu(\alpha))\}_{\alpha \in I}}$.

Let $A(*), B, \mu(\alpha)$, and $\lambda$ be as above. To prove $A(*) \rightarrow B$ it is enough to construct a family of pairs $(g(t), f(t))$ parametrized by $t \in \mathbb{C}^{*}$, where $f(t) \in I$ and $g(t) \in G L(V)$, with the following properties: Let $e_{1}, \ldots, e_{n}$ be a basis of $V$ and $c_{i, j}^{k}(1 \leq i, j, k \leq n)$ be the structure constants of $\lambda$ in this basis. If we construct $a_{i}^{j}: \mathbb{C}^{*} \rightarrow \mathbb{C}(1 \leq i, j \leq n)$ and $f: \mathbb{C}^{*} \rightarrow I$ such that $E_{i}^{t}=\sum_{j=1}^{n} a_{i}^{j}(t) e_{j}(1 \leq$ $i \leq n$ ) form a basis of $V$ for any $t \in \mathbb{C}^{*}$, and the structure constants of $\mu_{f(t)}$ in the basis $E_{1}^{t}, \ldots, E_{n}^{t}$ are such polynomials $c_{i, j}^{k}(t) \in \mathbb{C}[t]$ that $c_{i, j}^{k}(0)=c_{i, j}^{k}$, then $A(*) \rightarrow B$. In this case $E_{1}^{t}, \ldots, E_{n}^{t}$ and $f(t)$ are called a parametrized basis and a parametrized index for $A(*) \rightarrow B$ respectively.

Note also the following fact. Suppose that, for $\alpha \in \mathbb{C}$, the structure $\mu(\alpha) \in \mathfrak{L e i b}_{n}$ has structure constants $c_{i, j}^{k}(\alpha) \in \mathbb{C}$ in the basis $e_{1}, \ldots, e_{n}$, where $c_{i, j}^{k}(t) \in \mathbb{C}[t]$ for all $1 \leq i, j, k \leq n$. Let $X$ be some subset of $\mathfrak{L e i b}_{n}$ such that $\mu(\alpha) \in \bar{X}$ for $\alpha \in \mathbb{C} \backslash S$, where $S$ is a finite subset of $\mathbb{C}$. Then $\mu(\alpha) \in \bar{X}$ for all $\alpha \in \mathbb{C}$. Indeed, $\mu(\alpha) \in \overline{\{\mu(\beta)\}_{\beta \in \mathbb{C} \backslash S}} \subset \bar{X}$ for any $\alpha \in \mathbb{C}$. Thus, to prove that $\mu(\alpha) \in \bar{X}$ for all $\alpha \in \mathbb{C}$ we will prove that $\mu(\alpha) \in \bar{X}$ for all but finitely many $\alpha$.

Note that $A(*) \not \rightarrow B$ if $\operatorname{dim} \operatorname{Der}(A(\alpha))>\operatorname{dim} \operatorname{Der}(B)$ for all $\alpha \in I$, where $\operatorname{Der}(A)$ is the Lie algebra of derivations of $A$. Moreover, in the case of one algebra, if $A \rightarrow B$ and $A \neq B$, then $\operatorname{Der}(A)<\operatorname{Der}(B)$.

In other cases, the main tool for proving assertions of the form $A(*) \not \rightarrow B$ will be the following lemma.

Lemma 1 (Lemma 2 of [16]). Let $\mathcal{B}$ be a Borel subgroup of $G L(V)$ and $\mathcal{R} \subset \mathfrak{L e i b}_{n}$ be a $\mathcal{B}$-stable closed subset. If $A(*) \rightarrow B$ and for any $\alpha \in I$ the algebra $A(\alpha)$ can be represented by a structure $\mu(\alpha) \in \mathcal{R}$, then there is $\lambda \in \mathcal{R}$ representing $B$.

In all our applications of Lemma 1 we will use the group of lower triangular matrices as a Borel subgroup. This lemma can be applied in the case $|I|=1$ to prove non-degenerations. In particular, if $\operatorname{Ann}_{L}(A)>A n n_{L}(B), A^{2}<B^{2}$ or $A^{(+2)}<B^{(+2)}$, then $A \not \rightarrow B$ by Lemma1, We will use also the following two criteria that follow from Lemma 1. If $A \rightarrow B$, then the dimension of a maximal trivial 
subalgebra of $B$ is greater or equal to the dimension of a maximal trivial subalgebra of $A$ and the dimension of a maximal anticommutative subalgebra $D$ of $B$ such that $\operatorname{dim}(B D) \leq 1$ is greater or equal to the dimension of a maximal anticommutative subalgebra of $A$ satisfying the same condition.

In some cases we will construct the set $\mathcal{R}$ for the assertion $A(*) \not \rightarrow B$ explicitly. In this case we will define $\mathcal{R}$ as a set of structures $\mu$ satisfying some polynomial equations. In such a description we always denote by $c_{i j}^{k}(1 \leq i, j, k \leq n)$ the structure constants of $\mu$. Note also that in this case a condition of the form $S_{i} S_{j} \subset S_{k}$ is equivalent to a set of polynomial equations. Moreover, the set defined by such a condition is stable under the action of the group of lower triangular matrices.

\section{MAIN THEOREM}

The goal of this section is to describe irreducible components in $\mathfrak{L}_{\mathfrak{i} \mathfrak{i}} \mathfrak{b}_{4}$. The algebraic classification of four dimensional Lebniz algebras is based on the papers [1, 5, 21]. Let us give a brief introduction to this classification. The analogue of Levi-Malcev's theorem about the splitting of the solvable radical for Leibniz algebras was proved in [2]. Note that any semisimple (here this means that the solvable radical is zero) Leibniz algebra is a Lie algebra, and hence a Leibniz algebra is always the hemisemidirect product of a semisimple Lie algebra and a solvable ideal. The first step in the classification of four dimensional Leibniz algebras was done in [1], where all nilpotent four dimensional Leibniz algebras were classified. Then the description of all four dimensional solvable non-nilpotent Lebniz algebras was obtained in [5]. Finally, it was proved in [21] that there is only one non-solvable indecomposable Leibniz algebra whose dimension is less or equal to four, namely, the simple Lie algebra $\mathfrak{s l}_{2}$.

Based on the just mentioned works, we have constructed Table 1 that describes all four dimensional non-nilpotent non-Lie Leibniz algebras. Let us also introduce the following four dimensional Lie algebra structures:

$$
\begin{array}{llll}
\mathfrak{R}_{2} & e_{1} e_{2}=-e_{2} e_{1}=e_{2}, & e_{3} e_{4}=-e_{4} e_{3}=e_{4} ; \\
\mathfrak{s l}_{2} \oplus \mathbb{C} & e_{1} e_{2}=-e_{2} e_{1}=e_{2}, & e_{1} e_{3}=-e_{3} e_{1}=-e_{3}, \quad e_{2} e_{3}=-e_{3} e_{2}=e_{1} ; \\
g_{4}(a, b) & e_{1} e_{2}=-e_{2} e_{1}=e_{2}, & e_{1} e_{3}=-e_{3} e_{1}=e_{2}+a e_{3}, \quad e_{1} e_{4}=-e_{4} e_{1}=e_{3}+b e_{4} ; \\
g_{5}(a) & e_{1} e_{2}=-e_{2} e_{1}=e_{2}, & e_{1} e_{3}=-e_{3} e_{1}=e_{2}+a e_{3}, & \\
& e_{1} e_{4}=-e_{4} e_{1}=(a+1) e_{4}, & e_{2} e_{3}=-e_{3} e_{2}=e_{4} .
\end{array}
$$

Due to the results of [4], the variety of four dimensional Lie algebras contains 4 irreducible components, namely, $\overline{O\left(\mathfrak{s l}_{2} \oplus \mathbb{C}\right)}, \overline{O\left(\mathfrak{R}_{2}\right)}, \overline{\left\{O\left(g_{5}(a)\right)\right\}_{a \in \mathbb{C}}}$, and $\overline{\left\{O\left(g_{4}(a, b)\right)\right\}_{a, b \in \mathbb{C}}}$. In particular, there are 2 rigid four dimensional Lie algebras, namely, $\mathfrak{s l}_{2} \oplus \mathbb{C}$ and $\mathfrak{R}_{2}$.

The main result of the present paper is the following theorem.

Theorem 2. The variety $\mathfrak{L} \mathfrak{e i b}_{4}$ has 16 irreducible components:

$$
\begin{gathered}
\overline{\left\{O\left(g_{4}(a, b)\right)\right\}_{a, b \in \mathbb{C}}}, \overline{\left\{O\left(g_{5}(a)\right)\right\}_{a \in \mathbb{C}}}, \overline{\left\{O\left(\mathfrak{L}_{4}^{a}\right)\right\}_{a \in \mathbb{C}}}, \overline{\left\{O\left(\mathfrak{L}_{8}^{a}\right)\right\}_{a \in \mathbb{C}}}, \overline{\left\{O\left(\mathfrak{L}_{9}^{a}\right)\right\}_{a \in \mathbb{C}}}, \overline{\left\{O\left(\mathfrak{L}_{10}^{a}\right)\right\}_{a \in \mathbb{C}}}, \\
\overline{\left\{O\left(\mathfrak{L}_{15}^{a}\right)\right\}_{a \in \mathbb{C}}}, \overline{\left\{O\left(\mathfrak{L}_{18}^{a}\right)\right\}_{a \in \mathbb{C}}}, \overline{\left\{O\left(\mathfrak{L}_{21}^{a, b}\right)\right\}_{a, b \in \mathbb{C}}}, \overline{\left\{O\left(\mathfrak{L}_{22}^{a, b}\right)\right\}_{a, b \in \mathbb{C}}}, \overline{\left\{O\left(\mathfrak{L}_{23}^{a, b}\right)\right\}_{a, b \in \mathbb{C}}}, \\
\overline{O\left(\mathfrak{s l}_{2} \oplus \mathbb{C}\right)}, \overline{O\left(\mathfrak{R}_{1}\right)}, \overline{O\left(\mathfrak{R}_{2}\right)}, \overline{O\left(\mathfrak{R}_{3}\right)}, \overline{O\left(\mathfrak{L}_{44}\right)} .
\end{gathered}
$$


In particular, there are 5 rigid four dimensional Leibniz algebras:

$$
\mathfrak{s l}_{2} \oplus \mathbb{C}, \mathfrak{R}_{1}, \mathfrak{R}_{2}, \mathfrak{R}_{3}, \mathfrak{L}_{44} .
$$

Non-Lie algebras mentioned in the theorem are described in Table 1. The rest of the paper is devoted to the proof of Theorem 2 .

4.1. Nilpotent Leibniz algebras and conjectures about them. Several conjectures state that nilpotent Lie algebras form a very small subvariety in the variety of Lie algebras. Grunewald and O'Halloran conjectured in [11] that for any $n$-dimensional nilpotent Lie algebra $A$ there exists an $n$-dimensional non-nilpotent Lie algebra $B$ such that $B \rightarrow A$. At the same time, Vergne conjectured in [24] that a nilpotent Lie algebra cannot be rigid in the variety of all Lie algebras. Analogous assertions can be conjectured for other varieties. We will say that the variety $\mathfrak{A}$ of algebras has Grunewald-O'Halloran Property if for any nilpotent algebra $A \in \mathfrak{A}$ there is a non-nilpotent algebra $B \in \mathfrak{A}$ such that $B \rightarrow A$. We will say that $\mathfrak{A}$ has Vergne Property if there are no nilpotent rigid algebras in $\mathfrak{A}$. We will also say that $\mathfrak{A}$ has Vergne-Grunewald-O'Halloran Property if any irreducible component of $\mathfrak{A}$ contains a non-nilpotent algebra. Grunewald-O'Halloran Property was proved for four dimensional Lie and Jordan algebras in [4, 17] and for three dimensional Novikov and Leibniz algebras in [3,7]. Also, some results concerning Grunewald-O'Halloran Conjecture for Lie algebras were obtained in [12, 13].

It is clear that Vergne-Grunewald-O'Halloran Property follows from Grunewald-O'Halloran Property and Vergne Property follows from Vergne-Grunewald-O'Halloran Property. As a part of the proof of Theorem 2, we will show that $\mathfrak{L e i b}_{4}$ has Vergne-Grunewald-O'Halloran Property. On the other hand, we will show that the same variety does not have Grunewald-O'Halloran Property.

Let us also introduce the following four dimensional nilpotent Lebniz algebra structures:

$$
\begin{aligned}
& \mathfrak{N}_{3}^{a} \quad e_{1} e_{1}=e_{4}, \quad e_{1} e_{2}=a e_{4}, \quad e_{2} e_{1}=-a e_{4}, \quad e_{2} e_{2}=e_{4}, \quad e_{3} e_{3}=e_{4} ; \\
& \mathfrak{L}_{2}^{n} \quad e_{1} e_{1}=e_{2}, \quad e_{2} e_{1}=e_{3}, \quad e_{3} e_{1}=e_{4} ; \\
& \mathfrak{L}_{5}^{n} \quad e_{1} e_{1}=e_{3}, \quad e_{2} e_{1}=e_{3}, \quad e_{2} e_{2}=e_{4}, \quad e_{3} e_{1}=e_{4} \text {; } \\
& \mathfrak{L}_{11}^{n} \quad e_{1} e_{1}=e_{4}, \quad e_{1} e_{2}=-e_{3}, \quad e_{1} e_{3}=-e_{4}, \quad e_{2} e_{1}=e_{3}, \quad e_{2} e_{2}=e_{4}, \quad e_{3} e_{1}=e_{4} .
\end{aligned}
$$

It was proved in [16] that the variety of four dimensional nilpotent Leibniz algebras is formed by four irreducible components, namely, $\overline{O\left(\mathfrak{L}_{2}^{n}\right)}, \overline{O\left(\mathfrak{L}_{5}^{n}\right)}, \overline{O\left(\mathfrak{L}_{11}^{n}\right)}$ and $\overline{\left\{O\left(\mathfrak{N}_{3}^{a}\right)\right\}_{a \in \mathbb{C}}}$.

Lemma 3. Any irreducible component in $\mathfrak{L}_{\mathfrak{e} \mathfrak{i b}_{4}}$ contains a non-nilpotent algebra.

Proof. It is enough to prove that $\mathfrak{L}_{2}^{n}, \mathfrak{L}_{5}^{n}, \mathfrak{L}_{11}^{n}$ and $\mathfrak{N}_{3}^{a}$ belong to the closure of the union of orbits of non-nilpotent Lebniz algebras for any $a \in \mathbb{C}$. Let us prove this assertion case by case.

- The parametrized basis $E_{1}^{t}=t e_{1}+t e_{4}, E_{2}^{t}=t^{2} e_{2}+t^{2} e_{4}, E_{3}^{t}=t^{3} e_{3}+t^{3} e_{4}, E_{4}^{t}=-t^{4} e_{3}$ gives the degeneration $\mathfrak{L}_{40} \rightarrow \mathfrak{L}_{2}^{n}$.

- The parametrized basis $E_{1}^{t}=t e_{1}-t e_{2}+e_{3}, E_{2}^{t}=t^{2} e_{2}+e_{3}, E_{3}^{t}=t e_{3}-t^{3} e_{4}, E_{4}^{t}=t^{4} e_{4}$ with parametrized index $\epsilon(t)=-t$ give the assertion $\mathfrak{L}_{18}^{*} \rightarrow \mathfrak{L}_{5}^{n}$.

- The parametrized basis $E_{1}^{t}=t e_{1}-\frac{t}{2} e_{2}, E_{2}^{t}=\frac{t^{2}}{2} e_{2}+t^{2} e_{3}, E_{3}^{t}=t^{3} e_{3}-\frac{t^{3}}{4} e_{4}, E_{4}^{t}=\frac{t^{4}}{4} e_{4}$ with parametrized index $\epsilon(t)=\frac{t^{2}+1}{4}$ give the assertion $\mathfrak{L}_{15}^{*} \rightarrow \mathfrak{L}_{11}^{n}$. 
- The parametrized basis $E_{1}^{t}=t e_{1}+\frac{t}{2} e_{4}, E_{2}^{t}=i t e_{1}-i e_{3}-\frac{i t}{2} e_{4}, E_{3}^{t}=t e_{2}, E_{4}^{t}=t^{2} e_{4}$ gives the degeneration $\mathfrak{L}_{9}^{1+i a} \rightarrow \mathfrak{N}_{3}^{a}$ for any $a \in \mathbb{C}$.

Let us consider the degeneration $\mathfrak{L}_{40} \rightarrow \mathfrak{L}_{2}^{n}$ to clarify our proof. Writing nonzero products of $\mathfrak{L}_{40}$ in the basis $E_{i}^{t}$, we get

$$
E_{1}^{t} E_{1}^{t}=t^{2} e_{2}+t^{2} e_{4}=E_{2}^{t}, E_{2}^{t} E_{1}^{t}=t^{3} e_{3}+t^{3} e_{4}=E_{3}^{t}, E_{3}^{t} E_{1}^{t}=t^{4} e_{4}=t E_{3}^{t}+E_{4}^{t} .
$$

It is easy to see now that for $t=0$ we obtain the multiplication table of $\mathfrak{L}_{2}^{n}$. The remaining assertions can be considered in the same way.

Though we have proved that $\overline{O\left(\mathfrak{L}_{5}^{n}\right)}$ and $\overline{O\left(\mathfrak{L}_{11}^{n}\right)}$ are not irreducible components of $\mathfrak{L}_{\mathfrak{e} i \mathfrak{b}_{4}}$, we have not found an algebra that degenerates either to $\mathfrak{L}_{5}^{n}$ or to $\mathfrak{L}_{11}^{n}$ in the proof of Lemma 3 , In the proof of the next result we show that in fact there is no algebra that degenerates to $\mathfrak{L}_{5}^{n}$.

Theorem 4. $\mathfrak{L}_{\mathfrak{e i b}} \mathfrak{b}_{4}$ does not have Grunewald-O'Halloran Property.

Proof. To prove the theorem, we will show that there is no four dimensional Leibniz algebra that degenerates to $\mathfrak{L}_{5}^{n}$. Since $\mathfrak{L}_{5}^{n}$ is not a Lie algebra, only algebras from Table 1 can degenerate to it. Direct calculations show that the dimension of the Lie algebra of derivations is greater or equal to 3 for all algebras from Table 1 except $\mathfrak{R}_{1}$ and $\mathfrak{L}_{44}$. Since $\operatorname{dim} \operatorname{Der}\left(\mathfrak{L}_{5}^{n}\right)=3$ (see, for example [16]) it suffices to prove that $\mathfrak{R}_{1} \not \supset \mathfrak{L}_{5}^{n}$ and $\mathfrak{L}_{44} \not \rightarrow \mathfrak{L}_{5}^{n}$.

- $\mathfrak{R}_{1} \not \rightarrow \mathfrak{L}_{5}^{n}$ follows from the fact that $A n n_{L}\left(\mathfrak{R}_{1}\right)>A n n_{L}\left(\mathfrak{L}_{5}^{n}\right)$.

- To prove the assertion $\mathfrak{L}_{44} \not \rightarrow \mathfrak{L}_{5}^{n}$ let us consider the set

$\mathcal{R}=\left\{\begin{array}{l|c}\mu \in \mathfrak{L e i b}_{4} & S_{1} S_{3}+S_{4} S_{2}=0, S_{3} S_{2}+S_{4} S_{1} \subset S_{4}, S_{2} S_{2}+S_{3} S_{1} \subset S_{3}, S_{2} S_{1}+S_{1} S_{2} \subset S_{2}, \\ c_{12}^{2}+c_{21}^{2}=0, c_{31}^{3}=2 c_{21}^{2}, c_{12}^{3}=c_{21}^{3}\end{array}\right\}$.

It is not difficult to show that $\mathcal{R}$ is a closed subset of $\mathfrak{L e i b}_{4}$ that is stable under the action of the subgroup of lower triangular matrices and contains the structure $\mathfrak{L}_{44}$. It is also not difficult to show that $\mathcal{R} \cap O\left(\mathfrak{L}_{5}^{n}\right)=\varnothing$.

4.2. The proof of the main theorem. Now we are ready to prove Theorem 2. As a first step, we are going to prove that the irreducible components of the variety of four dimensional Lie algebras remain irreducible in $\mathfrak{L e i b}_{4}$. This fact follows from the next general lemma.

Lemma 5. Suppose that $A(*)=\{A(\alpha)\}_{\alpha \in T}$ is a set of $n$-dimensional non-Lie Leibniz algebras. If $B$ is an n-dimensional Lie algebra such that $A n n(B)=0$, then $A(*) \nrightarrow \rightarrow B$.

Proof. Since $A(\alpha)$ is non-Lie, the ideal $I(\alpha)=\{x y+y x \mid x, y \in A(\alpha)\}$ is nonzero for any $\alpha \in T$. Since $I(\alpha) \subset A n n_{L}(A(\alpha))$, we have $A n n_{L}(A(\alpha))>0=A n n(B)=A n n_{L}(B)$ for any $\alpha \in T$, and hence $A(*) \nrightarrow \rightarrow B$. 
It easily follows from Lemma 5 that if $\mathcal{C}$ is an irreducible component in the variety of $n$-dimensional Lie algebras containing an algebra with zero annihilator, then $\mathcal{C}$ is an irreducible component in $\mathfrak{L e i b}_{n}$. Indeed, suppose that $\mathcal{C}_{0}$ is an irreducible component of $\mathfrak{L e i b}_{n}$ containing $\mathcal{C}$. Let $\mathcal{L}$ be a set of all Lie

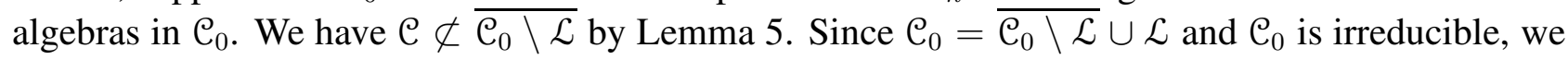
have $\mathcal{C}_{0}=\mathcal{L}$, and hence $\mathcal{C}_{0}=\mathcal{C}$.

Corollary 6. $\overline{O\left(\mathfrak{s l}_{2} \oplus \mathbb{C}\right)}, \overline{O\left(\mathfrak{R}_{2}\right)}, \overline{\left\{O\left(g_{5}(a)\right)\right\}_{a \in \mathbb{C}}}$ and $\overline{\left\{O\left(g_{4}(a, b)\right)\right\}_{a, b \in \mathbb{C}}}$ are irreducible components of $\mathfrak{L e i b}_{4}$.

Proof. The structure $\mathfrak{s l}_{2} \oplus \mathbb{C}$ is rigid in $\mathfrak{L e i b}_{4}$ as a unique non-solvable structure in the variety. The remaining part of the corollary follows from the fact that $\operatorname{Ann}\left(\mathfrak{R}_{2}\right)=0, \operatorname{Ann}\left(g_{5}(a)\right)=0$ for $a \neq-1$, and $\operatorname{Ann}\left(g_{4}(a, b)\right)=0$ for all $a, b \in \mathbb{C}^{*}$.

Proof of Theorem 2, Let $\mathcal{W}$ be the union of closed sets listed in the theorem. The assertions proved in Table 2 and the classification given in Table 1 show that all four dimensional non-nilpotent non-Lie Leibniz algebras belong to $\mathcal{W}$. All Lie algebras belong to $\mathcal{W}$ by the results of the paper [4]. Then it follows from Lemma 3 that all nilpotent Leibniz algebras belong to $\mathcal{W}$ too.

Thus, it remains to show that for any two different sets $X$ and $y$ listed in the theorem $X \not \subset y$. It follows from Corollary 6 and the fact that the set of four dimensional Lie algebras is a closed subset of $\mathfrak{L i b}_{4}$ that the required assertion is true if $X$ or $y$ is formed by Lie algebras.

Let us start with the algebras $\mathfrak{R}_{1}$ and $\mathfrak{R}_{3}$. These are the only two algebras from Table 1 that have two dimensional nilpotent radical. Since all other algebras have a three dimensional nilpotent radical, $\mathfrak{R}_{1}$ and $\mathfrak{R}_{3}$ do not belong to the closure of orbits of all the remaining structures of $\mathfrak{L}_{\mathfrak{e}} \mathfrak{b}_{4}$. Since $A n n_{L}\left(\mathfrak{R}_{1}\right)>A n n_{L}\left(\mathfrak{R}_{3}\right)$ and $\left(\mathfrak{R}_{3}\right)^{(+2)}<\left(\mathfrak{R}_{1}\right)^{(+2)}$, we have $\mathfrak{R}_{1} \not \rightarrow \mathfrak{R}_{3}$ and $\mathfrak{R}_{3} \not \rightarrow \mathfrak{R}_{1}$. Thus, $\overline{O\left(\mathfrak{R}_{1}\right)}$ and $\overline{O\left(\mathfrak{R}_{3}\right)}$ are irreducible components. Since $\operatorname{dim}\left(\mathfrak{R}_{1}\right)^{2}=\operatorname{dim}\left(\mathfrak{R}_{3}\right)^{2}=2$, these two components do not contain $\left\{\mathfrak{L}_{4}^{a}\right\}_{a \in \mathbb{C}},\left\{\mathfrak{L}_{8}^{a}\right\}_{a \in \mathbb{C}},\left\{\mathfrak{L}_{9}^{a}\right\}_{a \in \mathbb{C}},\left\{\mathfrak{L}_{10}^{a}\right\}_{a \in \mathbb{C}},\left\{\mathfrak{L}_{21}^{a, b}\right\}_{a, b \in \mathbb{C}},\left\{\mathfrak{L}_{22}^{a, b}\right\}_{a, b \in \mathbb{C}},\left\{\mathfrak{L}_{23}^{a, b}\right\}_{a, b \in \mathbb{C}}, \mathfrak{L}_{2}$, and $\mathfrak{L}_{44}$. Since $\operatorname{Ann}_{L}\left(\mathfrak{R}_{1}\right)>A n n_{L}\left(\mathfrak{L}_{15}^{a}\right)=\operatorname{Ann_{L}}\left(\mathfrak{L}_{18}^{a}\right)$ for any $a \neq 0$, we have $\mathfrak{R}_{1} \not \rightarrow \mathfrak{L}_{15}^{a}, \mathfrak{L}_{18}^{a}$ for $a \neq 0$. Note that $\mathfrak{R}_{3}$ contains a three dimensional anticommutative subalgebra $D=\left\langle e_{2}, e_{3}, e_{4}\right\rangle$ such that $\operatorname{dim}\left(\mathfrak{R}_{3} D\right)=1$. Since both $\mathfrak{L}_{15}^{a}$ and $\mathfrak{L}_{18}^{a}$ do not have such a subalgebra for any $a \neq 0$, we have $\mathfrak{R}_{3} \nrightarrow \rightarrow \mathfrak{L}_{15}^{a}, \mathfrak{L}_{18}^{a}$ for $a \neq 0$.

All the remaining algebras are solvable non-nilpotent Leibniz algebras with a three dimensional nilpotent radical. Moreover, one can check that each of them is represented by a structure $\mu$ such that $\left\langle e_{2}, e_{3}, e_{4}\right\rangle$ is the nilpotent radical and, moreover, the structure constants $c_{i j}^{k}(1 \leq i, j, k \leq 4)$ satisfy the conditions $c_{i j}^{k}=0$ if $i, j \geq 2$ and $k \leq \max (i, j)$ and $c_{1 i}^{j}=c_{i 1}^{j}=0$ for any $2 \leq$ $i, j \leq 4$ such that $j<i$. During this proof we will call a structure with three dimensional nilpotent radical that satisfies the described conditions a standard structure. Let us put in correspondence to a standard structure $\mu$ the 6 -tuple $S_{\mu}=\left(c_{21}^{2}, c_{12}^{2}, c_{31}^{3}, c_{13}^{3}, c_{41}^{4}, c_{14}^{4}\right) \in \mathbb{C}^{6}$. It is not difficult to show that if $S_{\mu}=\left(a_{1}, b_{1}, a_{2}, b_{2}, a_{3}, b_{3}\right)$ and $\lambda \in O(\mu)$ is a standard structure, then there is some permutation $\sigma:\{1,2,3\} \rightarrow\{1,2,3\}$ and some $c \in \mathbb{C}^{*}$ such that $S_{\lambda}=\left(c a_{\sigma(1)}, c b_{\sigma(1)}, c a_{\sigma(2)}, c b_{\sigma(2)}, c a_{\sigma(3)}, c b_{\sigma(3)}\right)$. Indeed, suppose that $\lambda$ and $\mu$ are standard structures and $\lambda=g^{-1} * \mu$ for some $g \in G L(V)$. Let us denote by $U$ the subspace $\left\langle e_{2}, e_{3}, e_{4}\right\rangle$ of $V$. Since $U$ is the nilpotent radical for $\mu$ and $\lambda$ at the 
same time, we have $g(U)=U$. We have also $g\left(e_{1}\right)=c e_{1}+u$ for some $c \in \mathbb{C}^{*}$, and $u \in U$. Let $L, R: U \rightarrow U$ denote the operators of the left and right multiplications by $e_{1}$ with respect to $\lambda$ correspondingly. Note that at the same time $L$ and $R$ are the operators of the left and right multiplications by $g\left(e_{1}\right)$ with respect to $\mu$ conjugated by $g$. Then it follows from the definition of a standard structure that, for any $s, t \in \mathbb{C}$, on one hand the characteristic polynomial $\chi_{s R+t L}(x) \in \mathbb{C}[x]$ of $s R+t L$ is $\prod_{i=1}^{3}\left(c\left(s a_{i}+t b_{i}\right)-x\right)$ and, on the other hand, the same polynomial is $\prod_{i=1}^{3}\left(s a_{i}^{\prime}+t b_{i}^{\prime}-x\right)$, where $S_{\lambda}=\left(a_{1}^{\prime}, b_{1}^{\prime}, a_{2}^{\prime}, b_{2}^{\prime}, a_{3}^{\prime}, b_{3}^{\prime}\right)$. The comparing of the roots of the obtained polynomials gives the required assertion.

Suppose now that $\left\{\mu_{\alpha}\right\}_{\alpha \in T}$ is a set of standard structures, $S_{\mu_{\alpha}}=\left(a_{1, \alpha}, b_{1, \alpha}, a_{2, \alpha}, b_{2, \alpha}, a_{3, \alpha}, b_{3, \alpha}\right)$, and the linear homogeneous polynomials $f_{1}, \ldots, f_{l} \in \mathbb{C}\left[x_{1}, x_{2}, x_{3}, x_{4}, x_{5}, x_{6}\right]$ are such that $f_{r}\left(a_{1, \alpha}, b_{1, \alpha}, a_{2, \alpha}, b_{2, \alpha}, a_{3, \alpha}, b_{3, \alpha}\right)=0$ for all $\alpha \in T$ and $1 \leq r \leq l$. If $\lambda \in \overline{\{O(\mu(\alpha))\}_{\alpha \in T}}$ is a standard structure with $S_{\lambda}=\left(a_{1}, b_{1}, a_{2}, b_{2}, a_{3}, b_{3}\right)$, then there is some permutation $\sigma:\{1,2,3\} \rightarrow$ $\{1,2,3\}$ and some $c \in \mathbb{C}^{*}$ such that $f_{r}\left(c a_{\sigma(1)}, c b_{\sigma(1)}, c a_{\sigma(2)}, c b_{\sigma(2)}, c a_{\sigma(3)}, c b_{\sigma(3)}\right)=0$ for all $1 \leq r \leq l$. Indeed, the conditions from the definition of a standard structure with the conditions $f_{r}\left(c_{21}^{2}, c_{12}^{2}, c_{31}^{3}, c_{13}^{3}, c_{41}^{4}, c_{14}^{4}\right)=0(1 \leq r \leq l)$ determine a closed subset of $\mathfrak{L e i b}_{4}$ that is invariant under the action of lower triangular matrices. Thus, it follows from Lemma 1 that $\lambda$ belongs to an orbit of some standard structure satisfying the equalities $f_{r}\left(c_{21}^{2}, c_{12}^{2}, c_{31}^{3}, c_{13}^{3}, c_{41}^{4}, c_{14}^{4}\right)=0(1 \leq r \leq l)$.

Computation of 6 -tuples for the structures under consideration gives the following results:

$$
\begin{array}{lll}
S_{\mathfrak{L}_{2}}=(1,-1,0,-1,1,0), & S_{\mathfrak{L}_{4}^{a}}=(1, a, a+1,-1,0,0), & S_{\mathfrak{L}_{8}^{a}}=(1, a, a+1,-1,-a, 0), \\
S_{\mathfrak{L}_{9}^{a}}=(1, a, 2,-1,-a, 0), & S_{\mathfrak{L}_{10}^{a}}=(1, a, 2,-1,0,0), & S_{\mathfrak{L}_{15}^{a}}=(0,1,0,0,-1,0), \\
S_{\mathfrak{L}_{18}^{a}}=(0,1,0,0,0,0), & S_{\mathfrak{L}_{21}^{a, b}}=(1, a, b,-1,-a, 0), & S_{\mathfrak{L}_{22}^{a, b}}=(1, a, b,-1,0,0), \\
S_{\mathfrak{L}_{23}^{a, b}}=(1, a, b, 0,0,0), & S_{\mathfrak{L}_{44}}=(1,2,3,-1,0,0) . &
\end{array}
$$

Almost all the required assertions follow now from our 6-tuple argument. Also the assertions of the form $A(*) \not \rightarrow B$, where $A(*) \in\left\{\mathfrak{L}_{21}^{*, *}, \mathfrak{L}_{22}^{*, *}, \mathfrak{L}_{23}^{*, *}\right\}$ and $B \in\left\{\mathfrak{L}_{2}, \mathfrak{L}_{4}^{a}, \mathfrak{L}_{8}^{a}, \mathfrak{L}_{9}^{a}, \mathfrak{L}_{10}^{a}, \mathfrak{L}_{15}^{a}, \mathfrak{L}_{18}^{a}, \mathfrak{L}_{44}\right\}_{a \in \mathbb{C}}$ follow from the fact that $A(a, b)$ has three dimensional trivial subalgebra for any $a, b \in \mathbb{C}$ and $B$ does not have three dimensional trivial subalgebra. Let us consider the remaining assertions case by case.

- $\mathfrak{L}_{4}^{*} \not \rightarrow \mathfrak{L}_{44}$. The required assertion follows from the fact that $\operatorname{Der}\left(\mathfrak{L}_{44}\right)>\operatorname{Der}\left(\mathfrak{L}_{4}^{a}\right)$ for any $a \in \mathbb{C}$.

- To prove the assertions $\mathfrak{L}_{9}^{*} \not \rightarrow \mathfrak{L}_{15}^{a}$ and $\mathfrak{L}_{10}^{*} \not \rightarrow \mathfrak{L}_{18}^{a}$ let us consider the set

$$
\mathcal{R}=\left\{\begin{array}{l|c}
\mu \in \mathfrak{L e i b}_{4} \mid \begin{array}{c}
S_{1} S_{1} \subset S_{2}, S_{2} S_{2} \subset S_{4}, S_{3} S_{1}+S_{1} S_{3} \subset S_{3}, S_{4} S_{1} \subset S_{4}, S_{1} S_{4}+S_{4} S_{2}=0, \\
c_{13}^{3}+c_{31}^{3}=0, c_{41}^{4}=2 c_{31}^{3}, c_{13}^{4}=c_{31}^{4}, c_{23}^{4}=c_{32}^{4}
\end{array}
\end{array}\right\} .
$$

It is not difficult to show that $\mathcal{R}$ is a closed subset of $\mathfrak{L e i b}_{4}$ that is stable under the action of the subgroup of lower triangular matrices and contains the structures $\mathfrak{L}_{9}^{b}$ and $\mathfrak{L}_{10}^{b}$ for any $b \in \mathbb{C}$. To see this it is enough to consider the basis $e_{1}, e_{3}, e_{2}, e_{4}$. It is also not difficult to show that $\mathcal{R} \cap O\left(\mathfrak{L}_{15}^{a}\right)=\mathcal{R} \cap O\left(\mathfrak{L}_{18}^{a}\right)=\varnothing$ for any $a \in \mathbb{C}$. 


\section{APPENDIX: TABLES}

Table 1. Four dimensional non-nilpotent non-Lie Leibniz algebras.

\begin{tabular}{|c|c|c|c|c|c|}
\hline $\mathfrak{R}_{1}$ & $e_{3} e_{1}=e_{3}$ & $e_{4} e_{2}=e_{4}$ & & & \\
\hline $\mathfrak{R}_{3}$ & $e_{2} e_{4}=-e_{4}$ & $e_{3} e_{1}=e_{3}$ & $e_{4} e_{2}=e_{4}$ & & \\
\hline $\mathfrak{L}_{2}$ & $e_{1} e_{1}=e_{4}$ & $e_{1} e_{2}=-e_{2}$ & $e_{1} e_{3}=e_{3}$ & $e_{2} e_{1}=e_{2}$ & \\
\hline & $e_{2} e_{3}=e_{4}$ & $e_{3} e_{1}=-e_{3}$ & $e_{3} e_{2}=-e_{4}$ & & \\
\hline $\mathfrak{L}_{4}^{a}$ & $e_{1} e_{2}=-e_{2}$ & $e_{2} e_{1}=e_{2}$ & $e_{3} e_{1}=a e_{3}$ & & \\
\hline & $e_{3} e_{2}=e_{4}$ & $e_{4} e_{1}=(1+a) e_{4}$ & & & \\
\hline $\mathfrak{L}_{5}$ & $e_{1} e_{1}=e_{4}$ & $e_{1} e_{2}=-e_{2}$ & $e_{2} e_{1}=e_{2}$ & $e_{3} e_{1}=-e_{3}$ & $e_{3} e_{2}=e_{4}$ \\
\hline $\mathfrak{L}_{6}$ & $e_{1} e_{1}=-e_{3}$ & $e_{1} e_{2}=-e_{2}$ & $e_{2} e_{1}=e_{2}+e_{4}$ & $e_{3} e_{2}=e_{4}$ & $e_{4} e_{1}=e_{4}$ \\
\hline $\mathfrak{L}_{7}$ & $e_{3} e_{1}=e_{3}$ & $e_{3} e_{2}=e_{4}$ & $e_{4} e_{1}=e_{4}$ & & \\
\hline $\mathfrak{L}_{8}^{a \neq-1,0}$ & $e_{1} e_{2}=-e_{2}$ & $e_{1} e_{3}=-a e_{3}$ & $e_{2} e_{1}=e_{2}$ & $e_{2} e_{3}=a e_{4}$ & \\
\hline & $e_{3} e_{1}=a e_{3}$ & $e_{3} e_{2}=e_{4}$ & $e_{4} e_{1}=(a+1) e_{4}$ & & \\
\hline $\mathfrak{L}_{9}^{a}$ & $e_{1} e_{2}=-e_{2}$ & $e_{1} e_{3}=-a e_{3}$ & $e_{2} e_{1}=e_{2}$ & $e_{2} e_{2}=e_{4}$ & \\
\hline & $e_{3} e_{1}=a e_{3}$ & $e_{4} e_{1}=2 e_{4}$ & & & \\
\hline $\mathfrak{L}_{10}^{a}$ & $e_{1} e_{2}=-e_{2}$ & $e_{2} e_{1}=e_{2}$ & $e_{2} e_{2}=e_{4}$ & $e_{3} e_{1}=a e_{3}$ & $e_{4} e_{1}=2 e_{4}$ \\
\hline $\mathfrak{L}_{11}$ & $e_{1} e_{1}=e_{3}$ & $e_{1} e_{2}=-e_{2}$ & $e_{2} e_{1}=e_{2}$ & $e_{2} e_{2}=e_{4}$ & $e_{4} e_{1}=2 e_{4}$ \\
\hline $\mathfrak{L}_{12}$ & $e_{1} e_{2}=-e_{2}$ & $e_{2} e_{1}=e_{2}$ & $e_{2} e_{2}=e_{4}$ & $e_{3} e_{1}=2 e_{3}+e_{4}$ & $e_{4} e_{1}=2 e_{4}$ \\
\hline $\mathfrak{L}_{13}$ & $e_{1} e_{2}=-e_{2}-e_{3}$ & $e_{1} e_{3}=-e_{3}$ & $e_{2} e_{1}=e_{2}+e_{3}$ & $e_{2} e_{2}=e_{4}$ & \\
\hline & $e_{3} e_{1}=e_{3}$ & $e_{4} e_{1}=2 e_{4}$ & & & \\
\hline $\mathfrak{L}_{14}$ & $e_{1} e_{3}=-e_{3}$ & $e_{2} e_{2}=e_{4}$ & $e_{3} e_{1}=e_{3}$ & & \\
\hline $\mathfrak{L}_{15}^{a}$ & $e_{1} e_{1}=a e_{4}$ & $e_{1} e_{2}=e_{4}$ & $e_{1} e_{3}=-e_{3}$ & $e_{2} e_{2}=e_{4}$ & $e_{3} e_{1}=e_{3}$ \\
\hline $\mathfrak{L}_{16}$ & $e_{1} e_{1}=-2 e_{4}$ & $e_{1} e_{3}=-e_{3}$ & $e_{2} e_{2}=e_{4}$ & $e_{3} e_{1}=e_{3}$ & \\
\hline $\mathfrak{L}_{17}$ & $e_{2} e_{2}=e_{4}$ & $e_{3} e_{1}=e_{3}$ & & & \\
\hline $\mathfrak{L}_{18}^{a}$ & $e_{1} e_{1}=a e_{4}$ & $e_{1} e_{2}=e_{4}$ & $e_{2} e_{2}=e_{4}$ & $e_{3} e_{1}=e_{3}$ & \\
\hline $\mathfrak{L}_{19}$ & $e_{1} e_{2}=e_{4}$ & $e_{2} e_{1}=e_{4}$ & $e_{2} e_{2}=e_{4}$ & $e_{3} e_{1}=e_{3}$ & \\
\hline $\mathfrak{L}_{21}^{a, b \neq 0}$ & $e_{1} e_{2}=-e_{2}$ & $e_{1} e_{3}=-a e_{3}$ & $e_{2} e_{1}=e_{2}$ & $e_{3} e_{1}=a e_{3}$ & $e_{4} e_{1}=b e_{4}$ \\
\hline $\mathfrak{L}_{22}^{a \neq 0, b \neq 0}$ & $e_{1} e_{2}=-e_{2}$ & $e_{2} e_{1}=e_{2}$ & $e_{3} e_{1}=a e_{3}$ & $e_{4} e_{1}=b e_{4}$ & \\
\hline $\mathfrak{L}_{23}^{a, b}$ & $e_{2} e_{1}=e_{2}$ & $e_{3} e_{1}=a e_{3}$ & $e_{4} e_{1}=b e_{4}$ & & \\
\hline $\mathfrak{L}_{24}^{a}$ & $e_{1} e_{1}=e_{4}$ & $e_{1} e_{2}=-e_{2}$ & $e_{1} e_{3}=-a e_{3}$ & $e_{2} e_{1}=e_{2}$ & $e_{3} e_{1}=a e_{3}$ \\
\hline $\mathfrak{L}_{25}^{a \neq 0}$ & $e_{1} e_{1}=e_{4}$ & $e_{1} e_{2}=-e_{2}$ & $e_{2} e_{1}=e_{2}$ & $e_{3} e_{1}=a e_{3}$ & \\
\hline $\mathfrak{L}_{26}^{a}$ & $e_{1} e_{1}=e_{4}$ & $e_{2} e_{1}=e_{2}$ & $e_{3} e_{1}=a e_{3}$ & & \\
\hline $\mathfrak{L}_{27}$ & $e_{1} e_{2}=-e_{2}$ & $e_{1} e_{3}=e_{4}$ & $e_{2} e_{1}=e_{2}$ & & \\
\hline $\mathfrak{L}_{28}$ & $e_{1} e_{3}=e_{4}$ & $e_{2} e_{1}=e_{2}$ & & & \\
\hline $\mathfrak{L}_{29}^{a \neq 0}$ & $e_{1} e_{2}=-e_{2}-e_{3}$ & $e_{1} e_{3}=-e_{3}$ & $e_{2} e_{1}=e_{2}+e_{3}$ & $e_{3} e_{1}=e_{3}$ & $e_{4} e_{1}=a e_{4}$ \\
\hline $\mathfrak{L}_{30}$ & $e_{1} e_{1}=e_{4}$ & $e_{1} e_{2}=-e_{2}-e_{3}$ & $e_{1} e_{3}=-e_{3}$ & $e_{2} e_{1}=e_{2}+e_{3}$ & $e_{3} e_{1}=e_{3}$ \\
\hline $\mathfrak{L}_{32}^{a}$ & $e_{2} e_{1}=e_{2}+e_{3}$ & $e_{3} e_{1}=e_{3}$ & $e_{4} e_{1}=a e_{4}$ & & \\
\hline $\mathfrak{L}_{33}$ & $e_{1} e_{1}=e_{4}$ & $e_{2} e_{1}=e_{2}+e_{3}$ & $e_{3} e_{1}=e_{3}$ & & \\
\hline
\end{tabular}




\begin{tabular}{l|lllll}
\hline $\mathfrak{L}_{34}^{a \neq 0}$ & $e_{1} e_{4}=-a e_{4}$ & $e_{2} e_{1}=e_{2}+e_{3}$ & $e_{3} e_{1}=e_{3}$ & $e_{4} e_{1}=a e_{4}$ & \\
\hline $\mathfrak{L}_{35}^{a \neq-1}$ & $e_{1} e_{2}=a e_{3}$ & $e_{1} e_{4}=-e_{4}$ & $e_{2} e_{1}=e_{3}$ & $e_{4} e_{1}=e_{4}$ & \\
\hline $\mathfrak{L}_{36}$ & $e_{1} e_{1}=e_{3}$ & $e_{1} e_{2}=-e_{3}$ & $e_{1} e_{4}=-e_{4}$ & $e_{2} e_{1}=e_{3}$ & $e_{4} e_{1}=e_{4}$ \\
\hline $\mathfrak{L}_{37}$ & $e_{1} e_{1}=e_{2}$ & $e_{1} e_{4}=-e_{4}$ & $e_{2} e_{1}=e_{3}$ & $e_{4} e_{1}=e_{4}$ & \\
\hline $\mathfrak{L}_{38}^{a}$ & $e_{1} e_{2}=a e_{3}$ & $e_{2} e_{1}=e_{3}$ & $e_{4} e_{1}=e_{4}$ & & \\
\hline $\mathfrak{L}_{39}$ & $e_{1} e_{1}=e_{3}$ & $e_{1} e_{2}=-e_{3}$ & $e_{2} e_{1}=e_{3}$ & $e_{4} e_{1}=e_{4}$ & \\
\hline $\mathfrak{L}_{40}$ & $e_{1} e_{1}=e_{2}$ & $e_{2} e_{1}=e_{3}$ & $e_{4} e_{1}=e_{4}$ & & \\
\hline $\mathfrak{L}_{41}$ & $e_{2} e_{1}=e_{2}+e_{3}$ & $e_{3} e_{1}=e_{3}+e_{4}$ & $e_{4} e_{1}=e_{4}$ & & \\
\hline $\mathfrak{L}_{44}$ & $e_{1} e_{2}=-e_{2}$ & $e_{2} e_{1}=e_{2}$ & $e_{2} e_{2}=e_{3}$ & $e_{3} e_{1}=2 e_{3}$ & \\
& $e_{3} e_{2}=e_{4}$ & $e_{4} e_{1}=3 e_{4}$ & & & \\
\end{tabular}

Table 2. Orbit closures for some families and degenerations of four dimensional Leibniz algebras.

\begin{tabular}{|c|c|c|c|c|c|c|c|c|}
\hline \multicolumn{3}{|l|}{ Assertions } & \multicolumn{5}{|c|}{ Parametrized bases } & \multirow{2}{*}{$\begin{array}{l}\text { Parametrized indices } \\
\epsilon(t)=t-1\end{array}$} \\
\hline $\mathfrak{L}_{8}^{*}$ & $\rightarrow$ & 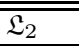 & $\overline{\overline{E_{1}^{t}}}$ & $=e_{1}+(t-1) e_{4}$, & $\overline{E_{2}^{t}=t e_{2},}$ & $\overline{E_{3}^{t}=e_{3},}$ & $\overline{E_{4}^{t}=(t-1) t e_{4}}$ & \\
\hline $\mathfrak{L}_{4}^{*}$ & $\rightarrow$ & $\mathfrak{L}_{5}$ & $E_{1}^{t}$ & $=e_{1}+e_{4}$ & $E_{2}^{t}=e_{2}$ & $E_{3}^{t}=t e_{3}$ & $E_{4}^{t}=t e_{4}$ & $\epsilon(t)=t-1$ \\
\hline $\mathfrak{L}_{4}^{+}$ & $\rightarrow$ & $\mathfrak{L}_{6}$ & $\frac{1}{E_{1}^{t}}$ & $=e_{1}-e_{3}$ & $E_{2}^{t}=e_{2}+e_{4}$ & $E_{3}^{t}=t e_{3}$ & $E_{4}^{t}=t e_{4}$ & $\epsilon(t)=t$ \\
\hline$\Re_{1}$ & $\rightarrow$ & $\mathfrak{L}_{7}$ & $E_{1}^{t}$ & $=e_{1}+e_{2}$ & $E_{2}^{t}=t e_{2}$ & $E_{3}^{t}=e_{3}+e_{4}$, & $E_{4}^{t}=t e_{4}$ & \\
\hline $\mathfrak{L}_{10}^{*}$ & $\rightarrow$ & $\mathfrak{L}_{11}$ & $\overrightarrow{E_{1}^{t}}$ & $=e_{1}+e_{3}$ & $E_{2}^{t}=e_{2}$ & $E_{3}^{t}=t e_{3}$ & $E_{4}^{t}=e_{4}$ & $\epsilon(t)=t$ \\
\hline $\mathfrak{L}_{10}^{*}$ & $\rightarrow$ & $\mathfrak{L}_{12}$ & $E_{1}^{t}$ & $=e_{1}$ & $E_{2}^{t}=t e_{2}$, & $E_{3}^{t}=e_{3}+e_{4}$, & $E_{4}^{t}=t^{2} e_{4}$ & $\epsilon(t)=2-t^{2}$ \\
\hline $\mathfrak{L}_{9}^{*}$ & $\rightarrow$ & $\mathfrak{L}_{13}$ & $E_{1}^{t}$ & $=e_{1}$ & $E_{2}^{t}=e_{2}+e_{3}$, & $E_{3}^{t}=t e_{3}$ & $E_{4}^{t}=e_{4}$ & $\epsilon(t)=t+1$ \\
\hline $\mathfrak{L}_{16}$ & $\rightarrow$ & $\mathfrak{L}_{14}$ & $E_{1}^{t}$ & $=e_{1}$ & $E_{2}^{t}=\frac{1}{t} e_{2}$, & $E_{3}^{t}=e_{3}$ & $E_{4}^{t}=\frac{1}{t^{2}} e_{4}$ & \\
\hline $\mathcal{L}_{15}^{*}$ & $\rightarrow$ & $\mathfrak{L}_{16}$ & $E_{1}^{t}$ & $=e_{1}$ & $E_{2}^{t}=\frac{1}{t} e_{2}$ & $E_{3}^{t}=e_{3}$, & $E_{4}^{t}=\frac{1}{t^{2}} e_{4}$ & $\epsilon(t)=-\frac{2}{t^{2}}$ \\
\hline $\mathfrak{R}_{1}$ & $\rightarrow$ & $\mathfrak{L}_{17}$ & $E_{1}^{t}$ & $=e_{1}$ & $E_{2}^{t}=t e_{2}+e_{4}$, & $E_{3}^{t}=e_{3}$ & $E_{4}^{t}=t e_{4}$ & \\
\hline $\mathfrak{L}_{18}^{*}$ & $\rightarrow$ & $\mathfrak{L}_{19}$ & $E_{1}^{t}$ & $=e_{1}+\frac{1}{t} e_{2}$, & $E_{2}^{t}=\frac{1}{t} e_{2}$ & $E_{3}^{t}=e_{3}$ & $E_{4}^{t}=\frac{1}{t^{2}} e_{4}$ & $\epsilon(t)=-\frac{1}{t^{2}}$ \\
\hline $\mathfrak{L}_{21}^{*, *}$ & $\rightarrow$ & $\mathfrak{L}_{24}^{a}$ & $E_{1}^{t}$ & $=e_{1}+e_{4}$ & $E_{2}^{t}=e_{2}$, & $E_{3}^{t}=e_{3}$, & $E_{4}^{t}=t e_{4}$ & $\epsilon(t)=(a, t)$ \\
\hline $\mathfrak{L}_{22}^{*, *}$ & $\rightarrow$ & $\mathfrak{L}_{25}^{a}$ & $\overrightarrow{E_{1}^{t}}$ & $=e_{1}+e_{4}$ & $E_{2}^{t}=e_{2}$ & $E_{3}^{t}=e_{3}$ & $E_{4}^{t}=t e_{4}$ & $\epsilon(t)=(a, t)$ \\
\hline $\mathfrak{L}_{23}^{*, *}$ & $\rightarrow$ & $\mathfrak{L}_{26}^{a}$ & $E_{1}^{t}$ & $=e_{1}+e_{4}$ & $E_{2}^{t}=e_{2}$ & $E_{3}^{t}=e_{3}$ & $E_{4}^{t}=t e_{4}$ & $\epsilon(t)=(a, t)$ \\
\hline $\mathfrak{R}_{3}$ & $\rightarrow$ & $\mathfrak{L}_{27}$ & $E_{1}^{t}$ & $=e_{2}+e_{3}$, & $E_{2}^{t}=e_{4}$ & $E_{3}^{t}=t e_{1}$, & $E_{4}^{t}=t e_{3}$ & \\
\hline $\mathfrak{R}_{1}$ & $\rightarrow$ & $\mathfrak{L}_{28}$ & $E_{1}^{t}$ & $=e_{1}+e_{4}$ & $E_{2}^{t}=e_{3}$ & $E_{3}^{t}=t e_{2}$ & $E_{4}^{t}=t e_{4}$ & \\
\hline $\mathfrak{L}_{21}^{*, *}$ & $\rightarrow$ & $\mathfrak{L}_{29}^{a}$ & $E_{1}^{t}$ & $=e_{1}$ & $E_{2}^{t}=e_{2}+e_{3}$ & $E_{3}^{t}=t e_{3}$ & $E_{4}^{t}=e_{4}$ & $\epsilon(t)=(t+1, a)$ \\
\hline $\mathfrak{L}_{29}^{*}$ & $\rightarrow$ & $\mathfrak{L}_{30}$ & $E_{1}^{t}$ & $=e_{1}+e_{4}$ & $E_{2}^{t}=e_{2}$ & $E_{3}^{t}=e_{3}$ & $E_{4}^{t}=t e_{4}$ & $\epsilon(t)=t$ \\
\hline $\mathfrak{L}_{23}^{*, *}$ & $\rightarrow$ & $\mathfrak{L}_{32}^{a}$ & $E_{1}^{t}$ & $=e_{1}$ & $E_{2}^{t}=e_{2}+e_{3}$, & $E_{3}^{t}=t e_{3}$, & $E_{4}^{t}=e_{4}$ & $\epsilon(t)=(t+1, a)$ \\
\hline $\mathfrak{L}_{26}^{*}$ & $\rightarrow$ & $\mathfrak{L}_{33}$ & $E_{1}^{t}$ & $=e_{1}$ & $E_{2}^{t}=e_{2}+e_{3}$, & $E_{3}^{t}=t e_{3}$, & $E_{4}^{t}=e_{4}$ & $\epsilon(t)=t+1$ \\
\hline $\mathfrak{L}_{22}^{*, *}$ & $\rightarrow$ & $\mathfrak{L}_{34}^{a}$ & $E_{1}^{t}$ & $=a e_{1}$, & $E_{2}^{t}=e_{3}+e_{4}$ & $E_{3}^{t}=t e_{4}$ & $E_{4}^{t}=e_{2}$ & $\epsilon(t)=\left(\frac{1}{a}, \frac{t+1}{a}\right)$ \\
\hline $\mathfrak{L}_{15}^{\frac{-a}{(1-a)^{2}}}$ & $\rightarrow$ & $\mathfrak{L}_{35}^{a \neq 1}$ & & $=e_{1}+\frac{1}{a-1} e_{2}$, & $E_{2}^{t}=t(a-1) e_{2}$ & $E_{3}^{t}=t e_{4}$ & $E_{4}^{t}=e_{3}$ & \\
\hline $\mathfrak{L}_{24}^{*}$ & $\rightarrow$ & $\mathfrak{L}_{36}$ & $E_{1}^{t}$ & $=t e_{1}$ & $E_{2}^{t}=e_{2}-t e_{4}$, & $E_{3}^{t}=t^{2} e_{4}$, & $E_{4}^{t}=e_{3}$ & $\epsilon(t)=\frac{1}{t}$ \\
\hline $\mathfrak{L}_{34}^{*}$ & $\rightarrow$ & $\mathfrak{L}_{37}$ & $E_{1}^{t}$ & $=t e_{1}+e_{2}$, & $E_{2}^{t}=t\left(e_{2}+e_{3}\right)$ & $E_{3}^{t}=t^{2} e_{3}$, & $E_{4}^{t}=e_{4}$ & $\epsilon(t)=\frac{1}{t}$ \\
\hline $\mathfrak{L}_{18}^{\frac{-a}{(1-a)^{2}}}$ & $\rightarrow$ & $\mathfrak{L}_{38}^{a \neq 1}$ & & $=e_{1}+\frac{1}{a-1} e_{2}$, & $E_{2}^{t}=t(a-1) e_{2}$, & $E_{3}^{t}=t e_{4}$, & $E_{4}^{t}=e_{3}$ & \\
\hline $\mathfrak{L}_{22}^{*, *}$ & $\rightarrow$ & $\mathfrak{L}_{39}$ & $E_{1}^{t}$ & $=t e_{1}+\frac{1}{t} e_{3}$, & $E_{2}^{t}=e_{2}-e_{3}$ & $E_{3}^{t}=t e_{3}$ & $E_{4}^{t}=e_{4}$ & $\epsilon(t)=\left(t, \frac{1}{t}\right)$ \\
\hline $\mathfrak{L}_{23}^{*, *}$ & $\rightarrow$ & $\mathfrak{L}_{40}$ & $E_{1}^{t}$ & $=t e_{1}+e_{2}+e_{3}$ & $E_{2}^{t}=t e_{2}+2 t e_{3}$ & $E_{3}^{t}=2 t^{2} e_{3}$ & $E_{4}^{t}=e_{4}$ & $\epsilon(t)=\left(2, \frac{1}{t}\right)$ \\
\hline $\mathfrak{L}_{23}^{*, *}$ & $\rightarrow$ & $\mathfrak{L}_{41}$ & $E_{1}^{t}$ & $=e_{1}$ & $E_{2}^{t}=e_{2}+2 e_{3}+e_{4}$ & $E_{3}^{t}=2 t\left(e_{3}+e_{4}\right)$, & $E_{4}^{t}=2 t^{2} e_{4}$ & $\epsilon(t)=(t+1,2 t+1)$ \\
\hline
\end{tabular}




\section{REFERENCES}

[1] Albeverio S., Omirov B., Rakhimov I., Classification of 4-dimensional nilpotent complex Leibniz algebras, Extracta Mathematicae, 21 (2006), 3, 197-210.

[2] Barnes D., On Levi's theorem for Leibniz algebras, Bulletin of the Australian Mathematical Society, 86 (2012), 184-185.

[3] Benes T., Burde D., Classification of orbit closures in the variety of three-dimensional Novikov algebras, Journal of Algebra and Its Applications, 13 (2014), 2, 1350081, 33 pp.

[4] Burde D., Steinhoff C., Classification of orbit closures of 4-dimensional complex Lie algebras, Journal of Algebra, 214 (1999), 2, 729-739.

[5] Cañete E., Khudoyberdiyev A., The classification of 4-dimensional Leibniz algebras, Linear Algebra and its Applications, 439 (2013), 1, 273-288.

[6] Calderon A., Camacho L., Omirov B., Leibniz algebras of Heisenberg type, Journal of Algebra, 452 (2016), 427447.

[7] Casas J., Khudoyberdiyev A., Ladra M., Omirov B., On the degenerations of solvable Leibniz algebras, Linear Algebra and its Applications, 439 (2013), 2, 472-487.

[8] Dherin B., Wagemann F., Deformation quantization of Leibniz algebras, Advances in Mathematics, 270 (2015), 21-48.

[9] Grunewald F., O’Halloran J., Varieties of nilpotent Lie algebras of dimension less than six, Journal of Algebra, 112 (1988), 315-325.

[10] Grunewald F., O'Halloran J., A Characterization of Orbit Closure and Applications, Journal of Algebra, 116 (1988), $163-175$.

[11] Grunewald F., O’Halloran J., Deformations of Lie algebras, Journal of Algebra, 162 (1993), 1, $210-224$.

[12] Herrera-Granada J.F., Tirao P., Filiform Lie algebras of dimension 8 as degenerations, Journal of Algebra and Its Applications, 13 (2014), 1350144.

[13] Herrera-Granada J.F., Tirao P., The Grunewald-O'Halloran Conjecture for Nilpotent Lie Algebras of Rank $\geq 1$, Communications in Algebra, 44 (2016), 5, 2180-2192.

[14] Kaygorodov I., Volkov Yu., The variety of 2-dimensional algebras over an algebraically closed field, Canadian Journal of Mathematics, 71 (2019), 4, 819-842.

[15] Kaygorodov I., Popov Yu., Volkov Yu., Degenerations of binary Lie and nilpotent Malcev algebras, Communications in Algebra, 46 (2018), 11, 4929-4941.

[16] Kaygorodov I., Popov Yu., Pozhidaev A., Volkov Yu., Degenerations of Zinbiel and nilpotent Leibniz algebras, Linear and Multilinear algebra, 66 (2018), 4, 704-716.

[17] Kashuba I., Martin M., Deformations of Jordan algebras of dimension four, Journal of Algebra, 399 (2014), 277289.

[18] Loday J.-L., Pirashvili T., Universal enveloping algebras of Leibniz algebras and (co)homology, Mathematische Annalen, 296 (1993), 1, 139-158.

[19] Mazzola G., The algebraic and geometric classification of associative algebras of dimension five, Manuscripta Mathematica, 27 (1979), 81-101.

[20] Mostovoy J., A comment on the integration of Leibniz algebras, Communications in Algebra, 41 (2013), 1, $185-194$.

[21] Omirov B., Rakhimov I., Turdibaev R., On Description of Leibniz Algebras Corresponding to $s l_{2}$, Algebras and Representation Theory, 16 (2013), 5, 1507-1519.

[22] Rakhimov I., Mohd Atan K., On Contractions and Invariants of Leibniz Algebras, Bulletin of the Malaysian Mathematical Sciences Society, 35 (2012), 557-565.

[23] Seeley C., Degenerations of 6-dimensional nilpotent Lie algebras over $\mathbb{C}$, Communications in Algebra, 18 (1990), 3493-3505.

[24] Vergne M., Cohomologie des algebres de Lie nilpotentes, Bulletin de la Société Mathématique de France, 98 (1970), $81-116$. 